\title{
Losartan Inhibits SARS-CoV-2 Replication in vitro
}

\author{
Reza Nejat ${ }^{1,2}$, Ahmad Shahir Sadr ${ }^{3,4}$, Brendan T. Freitas ${ }^{5}$, Jackelyn Murray ${ }^{6}$, Scott D. Pegan ${ }^{5}$, Ralph A. Tripp ${ }^{6}$, David J.
} Najafi $^{7}$

\begin{abstract}
${ }^{1}$ Department of Anesthesiology and Critical Care Medicine, Shahid Beheshti University of Medical Sciences, Tehran, Iran; ${ }^{2}$ ICU Chief, Bazarganan Hospital, Tehran, Iran; ${ }^{3}$ Bioinformatics Research Center, Cheragh Medical Institute and Hospital, Kabul, Afghanistan; ${ }^{4}$ Department of Computer and Data Sciences, Faculty of Mathematical Sciences, Shahid Beheshti University, Tehran, Iran; ${ }^{5}$ Department of Pharmaceutical and Biomedical Sciences, College of Pharmacy, University of Georgia, Athens, Georgia 30602; ${ }^{\circ}$ Department of Infectious Diseases, College of Veterinary Medicine, University of Georgia, Athens, Georgia , USA $;{ }^{7}$ Alliance Retinal Consultants, La Mesa California, USA
\end{abstract}

Corresponding author: David J. Najafi, Orcid ID: 0000-0001-5270-8922, Alliance Retina Consultants, 8262 University Ave, La Mesa, CA, 91942, USA; TEL: (619) 668-0045; email: dnajafimd@gmail.com

Received, March 30, 2021; Revised, June 30, 2021; Accepted, June 30, 2021; Published, July 27, 2021

\begin{abstract}
ABBREVIATION. ACE2: Angiotensin-Converting Enzyme II; ARB: angiotensin receptor blockers; AT1R: Angiotensin II type 1 receptor; AT2R: Angiotensin II type 2 receptor; DUB: deubiquitinase; PLpro: papain-like protease; proISG15: precursor ISG15; RAS: renin-angiotensin system; RBD: receptor binding domain; TLR: Toll-like receptor; Ub: ubiquitin; poly Ub: polyubiquitin
\end{abstract}

\begin{abstract}
Purpose: SARS-CoV-2 infection is associated with substantial mortality and high morbidity. This study tested the effect of angiotensin II type I receptor blocker, losartan, on SARS-CoV-2 replication and inhibition of the papain-like protease of the virus. Methods: The dose-dependent inhibitory effect of losartan, in concentrations from $1 \mu \mathrm{M}$ to $100 \mu \mathrm{M}$ as determined by quantitative cell analysis combining fluorescence microscopy, image processing, and cellular measurements (Cellomics analysis) on SARS-CoV-2 replication was investigated in Vero E6 cells. The impact of losartan on deubiquitination and deISGylation of SARS-CoV-2 papain-like protease (PLpro) were also evaluated. Results: Losartan reduced PLpro cleavage of tetraUbiquitin to diUbiquitin. It was less effective in inhibiting PLpro's cleavage of ISG15-AMC than Ubiquitin-AMC. To determine if losartan inhibited SARS-CoV-2 replication, losartan treatment of SARS-CoV-2 infected Vero E6 was examined. Losartan treatment one hour prior to SARS-CoV-2 infection reduced levels of SARS-CoV-2 nuclear protein, an indicator of virus replication, by $80 \%$ and treatment one-hour post-infection decreased viral replication by $70 \%$. Conclusion: Losartan was not an effective inhibitor of deubiquitinase or deISGylase activity of the PLpro but affected the SARS-CoV-2 replication of Vero E6 cells in vitro. As losartan has a favorable safety profile and is currently available it has features necessary for efficacious drug repurposing and treatment of COVID-19.
\end{abstract}

\section{INTRODUCTION}

COVID-19 pandemic caused by SARS-CoV-2 has created a global health and economic crisis. SARS$\mathrm{CoV}-2$ is an enveloped non-segmented singlestranded positive sense RNA virus belonging to the Coronaviridae family which includes SARS-CoV-1 and Middle East Respiratory Syndrome (MERS) coronavirus $[1,2]$. SARS-CoV-2 has $79 \%$ homology with SARS-CoV-1 at the genomic level [1-4].

COVID-19 is a multi-organ disease involving the lungs, cardiovascular system, gastrointestinal tract, and the central nervous system [5]. There is currently no effective antiviral drug, and the recently approved vaccines are not expected to be generally available globally [6]. Drug repurposing is a potential alternative strategy to restrict the disease. A drug that is globally available, safe, with beneficial properties in ameliorating the pathological changes of the disease and with minimum side-effects could drastically impact the management of this pandemic. SARS-CoV-2, like SARS-CoV-1, uses Angiotensin Converting Enzyme 2 (ACE2) to enter the host cells [7]. Attachment of the receptor binding domain (RBD) of S1 subunit of the spike protein (S protein) to ACE2 mediates the cell entry of the virus [8]. Upon SARS-CoV-2 entry into the host cell, replication is initiated through translation of two open reading frames, ORF1a and ORF1b, which produces two replicase polyproteins (ppla/pplab) containing non-structural proteins (nsps) contributing to its replication $[9,10]$. Autoproteolytic 
cleavage of $\mathrm{pp} 1 \mathrm{a} / \mathrm{pp} 1 \mathrm{ab}$ by cysteine proteases of the virus, papain-like protease (PLpro) and 3Chemotrypsin-like proteinase (3CLpro or Mpro), releases the nsps $[11,12]$. Having $83 \%$ homology with SARS-CoV-1 PLpro, this cysteine protease of SARS-CoV-2 has the ability to alter the intracellular microenvironment to promote viral replication. PLpros of these viruses post-translationally modify host cell proteins and immune mediators such as IFN- $\beta$, ISG15 protein, IRF3, TLR3, TLR4, TLR7 to evade innate immunity [13-23]. Drug-like noncovalent inhibitors of PLpros target these proteases for therapeutic purposes [24-29]. These types of inhibitors block PLpro cleavage of viral polypeptides as well as inhibition of its deubiqutinase and deISGylase functions [24-26, 30, 31].

Understanding the features of COVID-19 pathogenesis can aid in drug repurposing and discovery. Angiotensin receptor blockers (ARBs), including losartan, have been thought to be able to provide protection against COVID-19 pathogenesis [32-34]. It is thought that downregulation of ACE2, which occurs during SARS-CoV2 entry, leads to local RAS dysregulation with the increase in angiotensin II/angiotensin (1-7) ratio resulting in pro-inflammatory, pro-apoptotic and pro-thrombotic effects eventuating in COVID-19-induced cytokine storm [34-37]. In this context, human recombinant soluble ACE2 showed promising results in restricting the severe form of COVID-19, yet its short half-life requires further studies to prolong its circulation [38, 39]. Instead of increasing ACE2, selective AT1R antagonism by ARBs has been hypothesized to aid in ameliorating lung pathology in COVID-19 through rebalancing Ang II/angiotensin (1-7) ratio and indirectly promoting Ang II-induced activation of AT2R with antagonistic effect against Ang II activation of AT1R [34, 40]. A recent small randomized clinical trial showed that C21, an agonist of AT2R, could improve mortality rate and respiratory function significantly in hospitalized patients with COVID-19 [41]. However, the use of ARBs was constrained in the early phase of the pandemic due to concerns over their possible role in increasing viral load due to ACE2upregulating effect of these drugs [42, 43]. Further studies, on the other hand, have shown that ARBs are safe in patients with COVID-19 [44, 45]. This paved the way to conduct experimental and clinical studies on ARBs in COVID-19. An experimental study demonstrated that candesartan, an ARB, could decrease cytokine storm in COVID-19 [46]. In a clinical study on 75 hospitalized patients with COVID-19 who did not need intensive care revealed that candesartan could reduce hospital length of stay, time-to-negative swab test and time-to-improvement of chest X-ray [47]. Another recent multicenter randomized open-label controlled clinical trial on 158 patients showed that $80 \mathrm{mg}$ of telmisartan, another ARB, added to standard of care could improve biochemical and clinical indices with reducing CRP level, ICU admission rate and length of hospital stay compared to a control group [48]. In addition to its effect on RAS, losartan in a recent in silico study was shown to change the structure of ACE2 affecting its binding with the RBD of the SARS-CoV-2 S protein, hence, possibly decreasing the affinity of the virus to its receptor. Moreover, this study showed that losartan induces changes in atomic configuration of SARS-CoV-2 PLpro by occupying the place for inhibitors of PLpro with low docking energy (high affinity) (Fig. 1) [34]. In addition, based on the previous experimental studies, losartan attenuates the inflammatory responses leading to acute respiratory distress syndrome [49].

We sought to validate the bioinformatic findings in our previous in silico study [34] and examined the possible inhibitory effect of losartan against deubiquitinase and deISGylase properties of SARS-CoV-2 PLpro. We also examined if losartan could prevent viral replication of SARS-CoV-2 in pre- and post-infected Vero E6 cells.

\section{MATERIALS AND METHODS}

\section{Chemicals and Reagents}

Z-RLRGG-7-amino-4-methyl-courmarin (peptideAMC) was purchased from Bachem. Ubiquitin-7amino-4-methylcourmarin (Ub-AMC) was purchased from Boston Biochem; human ISG15-7amino-4-methylcourmarin (ISG15-AMC) was purchased from Boston Biochem. Lys48 linked tetra$\mathrm{Ub}$ were obtained from Boston Biochem; DLdithiothroitol (DTT) was purchased from GoldBio, and isopropyl- $\beta$-D-thiogalactopyranoside (IPTG) was purchased from GoldBio. 4-(2-Hydroxyethyl)1-piperazineethanesulfonic acid (HEPES) was purchased from Fisher BioReagents. Imidazole was purchased from Acros Organics; tris(hydroxymethyl)aminomethane (Tris) was purchased from Fisher Scientific. Losartan and Sodium chloride $(\mathrm{NaCl})$ were purchased from Fisher 


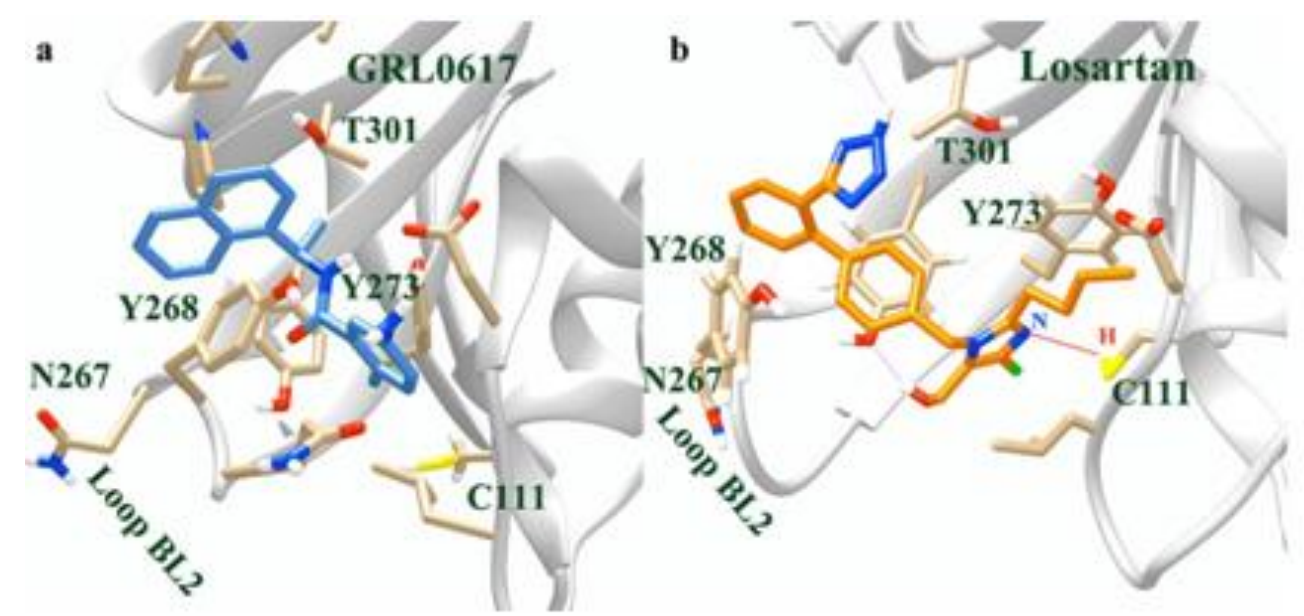

Fig. 1. Positioning of GRL-0617 (PLpro inhibitor) and losartan in the active site of PLpro. (a) X-ray crystallography structure of PLpro and GRL-0617 (PDB ID: 7cmd). (b) Losartan positioning was achieved after 100ns MD simulation. Losartan poses in the same position of the inhibitor (GRL-0617) according to PLpro amino acids in the circumferential area of these two ligands. Note: X-ray crystallography in contrast to NMR analysis and theoretical modeling cannot resolve hydrogen atoms in most protein crystals found in PDB files [50].

Chemical and bovine serum albumin (BSA) was purchased from Sigma Life Science.

\section{Determination of $\mathrm{IC}_{50}$ Values}

$\mathrm{IC}_{50}$ assays were performed using previously described methods for peptide-AMC cleavage experiments [51]. All assays were run using Corning Costar half-volume 96-well plates containing AMC buffer (100 mM NaCl, 50mM HEPES ( $\mathrm{pH}=7.5)$. $0.01 \mathrm{mg} / \mathrm{mL}$ bovine serum albumin (BSA), and 5 $\mathrm{mM}$ DTT) to a final volume of $50 \mu \mathrm{L}$ and performed in triplicate. The CLAIROstar plate reader (BMG Lab Tech, Inc.) was used to measure the fluorescence of the AMC cleavage, and the data was analyzed using MARS (BMG Lab Tech, Inc.). Assays using peptide-AMC substrate contained $1 \mu \mathrm{M}$ SARS-CoV2 PLpro and $50 \mu \mathrm{M}$ peptide-AMC in $98 \%$ AMC buffer $/ 2 \%$ DMSO. Reactions were performed in triplicate with inhibitor concentrations ranging from $390 \mathrm{nM}$ to $2.25 \mathrm{mM}$. Further assays utilizing ISG15AMC and Ub-AMC contained 1nM PLpro with 1 $\mu \mathrm{M}$ substrate or $25 \mathrm{nM}$ PLpro with $2 \mu \mathrm{M}$ substrate respectively.

\section{Inhibition of Poly-Ub and proISG15 Cleavage} Lys48 linked tetra-Ub obtained from Boston Biochem was incubated at $10.5 \mu \mathrm{M}$ with $23 \mathrm{nM}$ SARS-CoV-2 PLpro and $2.25 \mathrm{mM}$ losartan. Reactions were performed in $97.75 \%$ AMC buffer $/ 2.25 \%$ DMSO at a volume of $80 \mu \mathrm{L}$ and a temperature of $37^{\circ} \mathrm{C} .10 \mu \mathrm{L}$ samples were taken at the indicated time points and heat-shocked at $98^{\circ} \mathrm{C}$ for $5 \mathrm{~min}$. SDS-PAGE analysis was performed using Mini-PROTEAN TGX and Coomassie blue. Utilizing similar parameters $10 \mu \mathrm{M}$ human proISG15 was incubated with $20 \mathrm{nM}$ PLpro and $2 \mathrm{mM}$ losartan. Reactions were performed in $98 \%$ AMC buffer $/ 2 \%$ DMSO at a volume of $90 \mu \mathrm{L} .10 \mu \mathrm{L}$ samples were taken at the indicated time points and heat-shocked at $98^{\circ} \mathrm{C}$ for $5 \mathrm{~min}$. SDS-PAGE analysis was performed using Mini-PROTEAN TGX Stain-Free.

\section{SARS-CoV-2 and losartan Antiviral Assays.} SARS-CoV-2 (2019-nCoV/USA-WA1/2020; MN985325.1) was received from BEI resources and propagated in Vero E6 cells. SARS-CoV-2 studies were performed in an approved BSL3 facility in the Animal Health Research Center (AHRC) at the University of Georgia. Work followed Biosafety in Microbiological and Biomedical Laboratories (BMBL) guidelines Appendix F5 from the CDC. Infection was done at a multiplicity of infection $(\mathrm{MOI})=0.1$ in serum-free in Dulbecco's minimal essential medium (DMEM) for $1 \mathrm{~h}$ after which the virus-containing media was decanted and replaced with DMEM supplemented with $1 \%$ heat-inactivated fetal bovine serum [52]. The virus was propagated for $56 \mathrm{~h}$ before it was harvested, and the titer determined by plaque assay on Vero E6 cells [53]. The viral plaques were counted, and the titer was determined as PFU/ml. The Vero cells were plated at $2 \times 10^{4}$ cells/well in a 96-well plate and incubated overnight at $37^{\circ} \mathrm{C}$. Losartan was prepared in DMEM to $100 \mu \mathrm{M}, 50 \mu \mathrm{M}, 25 \mu \mathrm{M}, 12 \mu \mathrm{M}, 6 \mu \mathrm{M}, 3 \mu \mathrm{M}$ or 1 
$\mu \mathrm{M}$. The 96-well plate was washed with PBS and the dilutions of losartan added in triplicate. The cells were preincubated for 1 hour, the plate was washed once with PBS and then infected at a MOI $=0.01$ for $1 \mathrm{~h}$ after which the virus containing media was removed and fresh losartan concentrations were added to the cells in a final volume of $200 \mu \mathrm{L}$ per well and incubated for $96 \mathrm{~h}$ at $37^{\circ} \mathrm{C}$ at $5 \% \mathrm{CO} 2$. Otherwise, cells were plated at $2 \times 10^{4}$ cells/well in a 96-well plate, then infected at a MOI $=0.01$ for $1 \mathrm{~h}$ after which the virus containing media was removed, and media containing $200 \mu \mathrm{L}$ of diluted losartan added per well and incubated for $96 \mathrm{~h}$ in triplicate. These plates were fixed, stained for viral nuclear protein (NP) levels and assayed on a Cellomics ArrayScan. Levels of NP correlates with replication the infected cells [54]. Average percentage responder intensity of the fluorescent channel from NPs of the virus was measured and the calculations were performed using Prism 8 from GraphPad.

\section{Statistical Analysis}

Statistical analysis was performed with GraphPad Prism 8.0. $\mathrm{IC}_{50}$ and $\mathrm{EC}_{50}$ calculations and statistical analysis were performed using Prism 8.0 from GraphPad. P-values $<0.001$ are statistically significant. Comparisons between two normally distributed groups were performed by Wilcoxon signed rank test using Prism 8.

\section{RESULTS}

\section{Deubiquitinase and deISGylase inhibitory activity of losartan on SARS-CoV-2 PLpro.}

To characterize its inhibitory effects on SARS-CoV2 PLpro, losartan was incubated at various concentrations with SARS-CoV-2 PLpro and a peptide substrate containing the last five consensus amino acids of ubiquitin (Ub) and interferon stimulated gene product 15 (ISG15) in conjugation with a C-terminal 7-amido-4-methylcoumarin (AMC) fluorogenic reporter group (peptide-AMC). These assays revealed $\mathrm{IC}_{50}$ value of $1200 \pm 61 \mu \mathrm{M}$ for losartan against peptide-AMC (Table 1). ISG15 is the preferred substrate of CoV2 PLpro [24]. To test its inhibitory effect, losartan was investigated against ISG15-AMC and peptideAMC. SARS-CoV-2 PLpro has a strong preference for ISG15 over peptide as a substrate displacing competitive inhibitors with lower affinity for ISG15. These data suggest that losartan, due to its structure, may be interacting with elements of PLpro that accommodate both peptide and Ub-like substrates such as the active site, or the P3/P4 pocket of the PLpro [26]. This pocket is adjacent to the active site and facilitates cleavage of Ubl substrates by binding the Cterminal leucine and arginine of the RLRGG motif [26]. Other small molecule PLpro inhibitors of similar size have been shown to bind in this pocket in both SARS-CoV-1 and SARS-CoV-2 [25].

\section{Efficacy of losartan for Inhibiting CoV2 PLpro.}

To explore the relationship between substrate affinity and inhibitor efficacy, losartan was tested against Ub-AMC at $200 \mu \mathrm{M}$. SARS-CoV2 PLpro has a strong preference for ISG15 over Ub as a substrate [24], where the catalytic efficiency of the PLpro for Ub is approximately one-tenth that of ISG15 but more than 250 times that of the peptideAMC. Therefore, competitive non-covalent inhibitors limit Ub-AMC cleavage at a rate somewhere between those of ISG15-AMC and peptide-AMC. Losartan had an inhibition rate of $2.3 \%$ when tested against Ub-AMC (Table 1). Inhibition rate of losartan was $6.9 \%$ against ISG15 cleavage (Table 1). These differences between inhibitory rates of losartan against ISG15-AMC and Ub-AMC are unlikely to have a qualitative difference in a biological system. To confirm this, molecular weight shift assays were performed with more biologically accurate substrates.

\section{Effect of losartan on Tetra-Ub Deubiqutination.}

Ubl substrates with AMC tags at their C-terminal glycine are not entirely reflective of natural substrates. Also, PLpros have been observed to prefer cleaving poly-Ub chains over mono-Ub substrates [55]. To determine if the inhibitory values derived from AMC cleavage assays are reflective of a qualitative change in DUB and deISGylase activity, losartan was tested against human proISG15 and K48 linked tetra-Ub. As expected, losartan at $2 \mathrm{mM}$ shows a small reduction deISGylase activity relative to the control (Fig. 2). However, losartan at $2.25 \mathrm{mM}$ effectively eliminated DUB activity (Fig. 3).

Activity of losartan on SARS-CoV-2 replication. Losartan treatment of Vero E6 cells showed a dosedependent $(0-100 \mu \mathrm{M}$ concentrations $)$ and significant $(\mathrm{p}<0.001)$ reduction in SARS-CoV-2 replication in both pre- and post-infection studies (Fig. 4). The half- maximal effective concentration 
Table 1. Percentile of inhibition and $\mathrm{IC}_{50}$ of cleavage of peptide-AMC, percentile of inhibition of ISG15-AMC and Ub-AMC in the presence of $200 \mu \mathrm{M}$ losartan. IC $_{50}$ of losartan for cleavage of peptide-AMC is $1200 \pm 61$ $\mu \mathrm{M}$. IC $\mathrm{C}_{50}$ of ISG15 and $\mathrm{Ub}$ were not determined needing high concentrations of losartan

\begin{tabular}{|c|c|c|c|c|}
\hline & Peptide $(200 \mu \mathrm{M})$ & PeptideIC $_{50}(\mu \mathrm{M})$ & ISG15 $(200 \mu M)$ & Ub $(200 \mu M)$ \\
\hline Losartan & $35.1 \% \pm 2.7$ & $1200 \pm 61$ & $6.9 \% \pm 3.4$ & $2.3 \% \pm 1.4$ \\
\hline
\end{tabular}

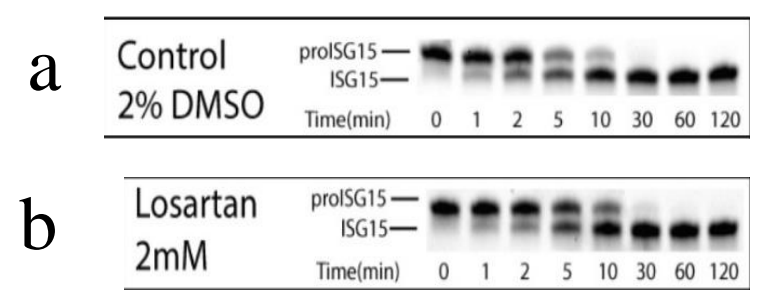

Fig. 2. Gel shift analysis. Inhibitory activity of losartan on deISGylation of PLpro. (a) deISGylation of PLpro on proISG15 in $10 \mu \mathrm{M}$ of proISG run against $20 \mathrm{nM}$ of SARS-CoV-2 PLpro, at $37^{\circ} \mathrm{C}$, over 2 hours with samples taken at the indicated intervals with Gel cleavage assay visualized by Commassie Blue Staining. Strong PLpro deISGylation is noted. (b) Losartan, at lower concentrations up to $2 \mathrm{mM}$ concentration added to proISG15 and PLpro solution, demonstrates insignificant inhibitory effect on PLpro deISGylation between 5 and 30 minutes.

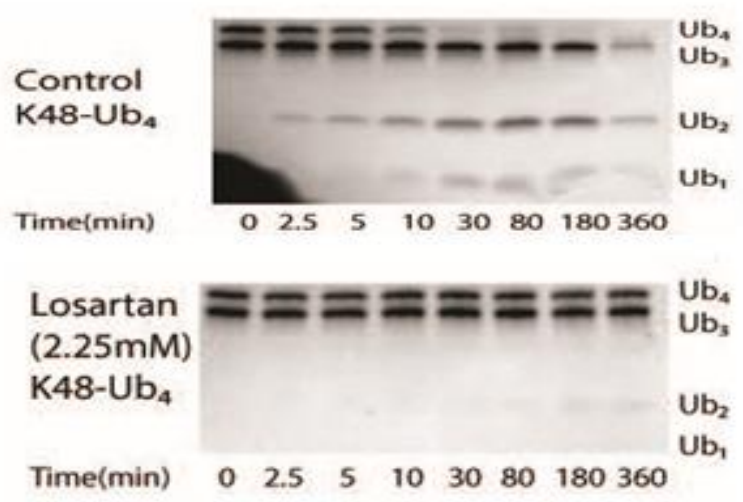

Fig. 3. Inhibitory effect of losartan on deubiquitination activity of PLpro on $\mathrm{K} 48-\mathrm{Ub}_{4}$ (a) At the mentioned intervals over 6 hours, multiple ten $\mu \mathrm{L}$ samples of Lys 48 linked tetra $\mathrm{Ub}$ at $13.65 \mu \mathrm{M}$ with $23 \mathrm{nM} \mathrm{CoV} 2$ PLpro in AMC buffer at $37{ }^{\circ} \mathrm{C}$ were taken and heat-shocked at 98 ${ }^{\circ} \mathrm{C}$ for 5 minutes. Gel cleavage assay visualized by Commassie Blue Staining shows PLpro's cleavage of $\mathrm{Ub}_{4}$ to mostly DUB and some monoUb. (b) Addition of losartan, at $2.25 \mathrm{mM}$ concentration, demonstrates a significant inhibitory effect on PLpro deUbiquitination of $\mathrm{Ub}_{4}$. Due to the low inhibitory effect of losartan on $\mathrm{Ub}$
(2.3\% at $200 \mu \mathrm{M}$ in Table 1) $2.25 \mathrm{mM}$ was used in this experiment.

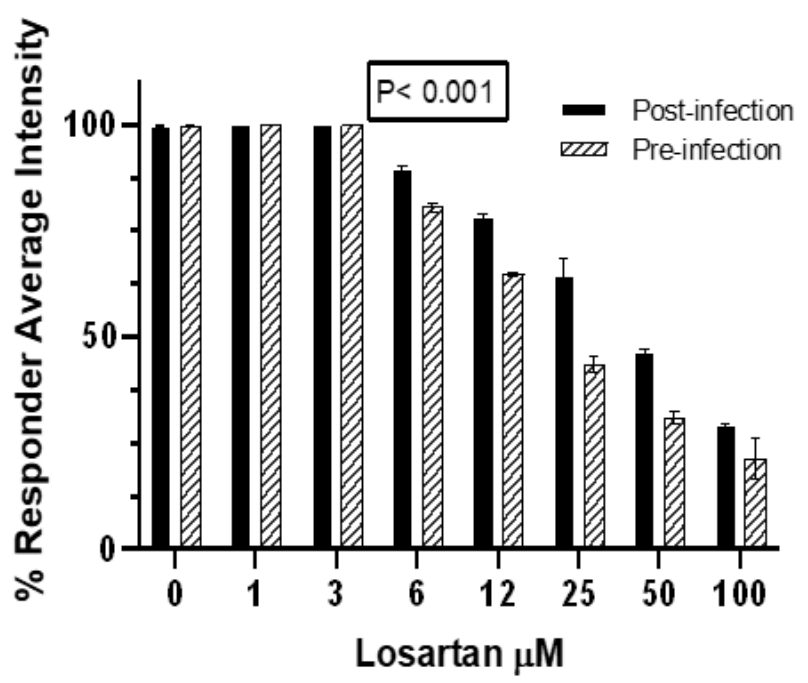

Fig. 4. Losartan treatment, in a dose-dependent manner (1$100 \mu \mathrm{M})$, decreases CoV2-infected Vero E6 cells preinfection more significantly than post-infection (Wilcoxon test $\mathrm{p}<0.001)$.

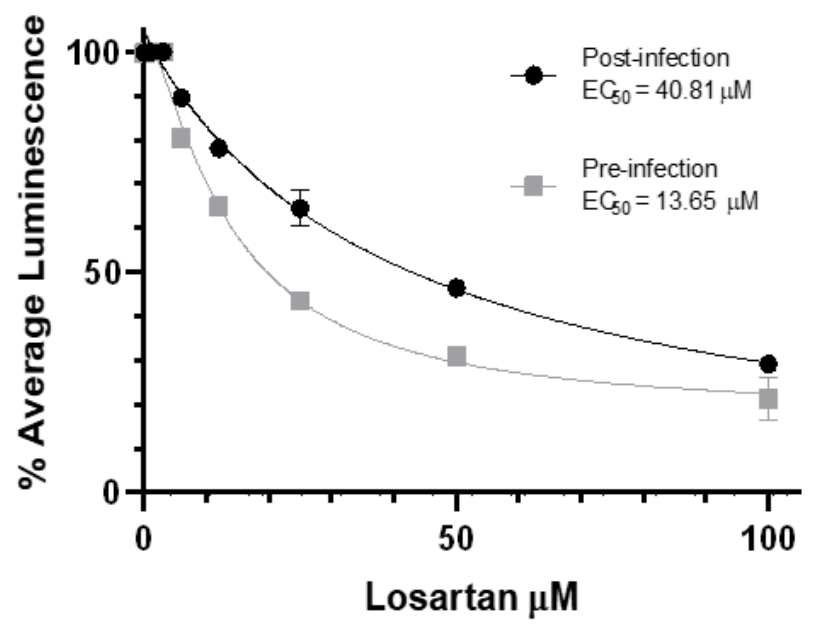

Fig. 5. SARS-CoV-2 replication in pre- and post-infection treatment. Losartan dose-dependently reduces the production of viral nuclear proteins in Vero E6 cells $(80 \%) . \mathrm{EC}_{50}$ curves are significantly different in pre- and post-infection groups $(\mathrm{P}<0.001)$. 
$\left(\mathrm{EC}_{50}\right)$ of losartan significantly $(\mathrm{p}<0.001)$ decreased SARS-CoV-2 replication using $41 \mu \mathrm{M}$ in postinfection treatment and $13.7 \mu \mathrm{M}$ in the pre-infection treatment (Fig. 5).

\section{DISCUSSION}

Replication of SARS-CoV-2 depends on PLpro and Mpro functions [31]. Inhibitors of SARS-CoV-1 PLpro, in several studies, have been shown to be as effective against SARS-CoV-2 PLpro [56, 57]. Although many of the findings were promising, the development in introducing approved drugs is lagging. Our in vitro study revealed that losartan was a weak inhibitor of PLpro in cleavage of peptideAMC $\left(\mathrm{IC}_{50}=1200 \pm 61 \mu \mathrm{M}\right)$. It did not substantially suppress deISGylating ability of PLpro (Fig. 3). It is possible that losartan interacts with the $\mathrm{P} 3 / \mathrm{P} 4$ pocket of PLpro adjacent to its active site and is displaced in the presence of ISG15. This pocket could facilitate cleavage of Ubl substrates by binding the $\mathrm{C}$ terminal of leucine and arginine, of the RLRGG motif, through a protonating reaction in a cleft in the PLpro containing a conserved triad of Histidine(H272)-Cysteine(C111)-Aspartic Acid(D286) in papain-like proteases [26, 58, 59].

It has been reported that SARS-CoV-2 PLpro prefers deISGylation ten times more than deubiquitination [24]. However, our study demonstrated that losartan in $2.25 \mathrm{mM}$ concentration could completely suppress cleavage of K48 linked polyubiquitin chains by PLpro (Fig. 4). This underscores that due to PLpro substrate affinity, a PLpro's viral evasion mechanism facilitated by their deubiquitinase activities may be impacted at lower competitive inhibitor concentrations than that of their deISGylase activities.

Considering the putative effects of losartan in the treatment of COVID-19, we assessed its impact on SARS-CoV-2 replication in Vero E6 cells. Vero E6 cells are derived from the kidney of African green monkeys and express ACE2 [60-62]. These cells do not produce IFN type I allowing SARS-CoV-1 and SARS-CoV-2 replication without IFN inhibition [6367]. In our study, losartan treatment prevented SARS-CoV-2 replication. At the highest losartan concentrations, pre-infection and post-infection treatment prevented SARS-CoV-2 replication by $80 \%$ and $70 \%$, respectively (Figures 5 and 6). Based on $\mathrm{EC}_{50}$, when used prior to infecting the cells (preinfection), losartan with an EC50 of $13.6 \mu \mathrm{M}$ reduced SARS-CoV-2 replication by more than $50 \%$ compared to post-infection treatment with this drug with an $\mathrm{EC}_{50}$ of $40.8 \mu \mathrm{M}$. The effect of losartan in pre-infection is in agreement with a previously published analysis showing SARS-CoV-2 had a lower affinity for ACE2 in the presence of losartan [34].

In addition to our experimental study ongoing clinical trials are assessing the efficacy of losartan in the treatment of COVID-19. Data from future robust clinical trials combined with the data from this study will validate whether globally available and inexpensive ARBs could improve the management of COVID-19.

\section{CONCLUSION}

Losartan showed weak inhibitory effect on viral PLpro deubiquitinase and deISGylase properties. Importantly, losartan treatment of Vero E6 cells prior to and after SARS-CoV-2 infection inhibited the virus replication. Additional experiments are needed to better assess the structural changes in the viral proteins and their biological products when exposed to losartan. Outcome of these experiments may provide a viable pathway for antiviral design and development. Ongoing and future randomized clinical trials in different countries with well diversified cohort would significantly aid in our understanding the effect of losartan on the course of COVID-19. Losartan with its in vitro inhibitory effect on viral replication and its significant benefits in cell protection without cell toxicity may play an important role in slowing the spread of the disease, medical management of individuals unresponsive to vaccination or new mutations, easing healthcare cost and burden in communities globally.

AUTHOR CONTRIBUTIONS. The manuscript was written through the contributions of all authors. All authors have approved the final version of the manuscript.

Viral studies were performed at the Animal Health Research Center (AHRC) of the University of Georgia, an approved BSL3 facility, under the supervision of RT.

\section{CONFLICTS AND FINANCIAL INTERESTS}

No conflict of interest to report.

ACKNOWLEDGMENTS. We thank our families and friends for their support which provided us with the time to pursue this endeavor while they endured the challenges that all families have encountered in this pandemic. 
This work was supported by the University of Georgia College Of Pharmacy, College of Veterinary Medicine, and the University of Georgia's Center for Drug Discovery and the Georgia Research Alliance.

We thank Derren Barken, PhD for helping with verification of our statistical analysis of the data presented in this research.

We thank California Medical Innovations Institute for their reception and encouragement.

\section{REFERENCES}

1. Chan, J.F.-W., et al., Genomic characterization of the 2019 novel humanpathogenic coronavirus isolated from a patient with atypical pneumonia after visiting Wuhan. Emerging microbes \& infections, 2020. 9(1): p. 221-236.

2. Gorbalenya, A., et al., The species severe acute respiratory syndrome related coronavirus: classifying 2019-nCoV and naming it SARS-CoV-2. . Nat Microbiol, 2020(5): p. 536-544.

3. Rossi, G.A., et al., Differences and similarities between SARS-CoV and SARS-CoV-2: spike receptor-binding domain recognition and host cell infection with support of cellular serine proteases. Infection, 2020: p. 1-5.

4. Lu, R., et al., Genomic characterisation and epidemiology of 2019 novel coronavirus: implications for virus origins and receptor binding. The Lancet, 2020. 395(10224): p. 565-574.

5. Gavriatopoulou, M., et al., Organ-specific manifestations of COVID-19 infection. Clinical and experimental medicine, 2020: p. 1-14.

6. Mullard, A., How COVID vaccines are being divvied up around the world. Nature, 2020.

7. $\mathrm{Ou}, \mathrm{X}$., et al., Characterization of spike glycoprotein of SARS-CoV-2 on virus entry and its immune cross-reactivity with SARSCoV. Nature communications, 2020. 11(1): p. 1-12.

8. Huang, Y., et al., Structural and functional properties of SARS-CoV-2 spike protein: potential antivirus drug development for COVID-19. Acta Pharmacologica Sinica, 2020. 41(9): p. 1141-1149.

9. Helmy, Y.A., et al., The COVID-19 pandemic: a comprehensive review of taxonomy, genetics, epidemiology, diagnosis, treatment, and control. Journal of Clinical Medicine, 2020. 9(4): p. 1225.

10. Fehr, A.R. and S. Perlman, Coronaviruses: an overview of their replication and pathogenesis, in Coronaviruses. 2015, Springer. p. 1-23.

11. Snijder, E., E. Decroly, and J. Ziebuhr, The nonstructural proteins directing coronavirus RNA synthesis and processing, in Advances in virus research. 2016, Elsevier. p. 59-126.

12. Astuti, I., Severe Acute Respiratory Syndrome Coronavirus 2 (SARS-CoV-2): An overview of viral structure and host response. Diabetes \& Metabolic Syndrome: Clinical Research \& Reviews, 2020.

13. Devaraj, S.G., et al., Regulation of IRF-3dependent innate immunity by the papain-like protease domain of the severe acute respiratory syndrome coronavirus. Journal of Biological Chemistry, 2007. 282(44): p. 32208-32221.

14. Xing, Y., et al., The papain-like protease of porcine epidemic diarrhea virus negatively regulates type I interferon pathway by acting as a viral deubiquitinase. The Journal of general virology, 2013. 94(Pt 7): p. 1554.

15. Bailey-Elkin, B.A., et al., Crystal structure of the Middle East respiratory syndrome coronavirus (MERS-CoV) papain-like protease bound to ubiquitin facilitates targeted disruption of deubiquitinating activity to demonstrate its role in innate immune suppression. Journal of Biological Chemistry, 2014. 289(50): p. 34667-34682.

16. Mielech, A.M., et al., Murine coronavirus ubiquitin-like domain is important for papainlike protease stability and viral pathogenesis. Journal of virology, 2015. 89(9): p. 49074917.

17. Mielech, A.M., et al., Nidovirus papain-like proteases: multifunctional enzymes with protease, deubiquitinating and deISGylating activities. Virus research, 2014. 194: p. 184190.

18. Nejat, R. and A.S. Sadr, SARS virus papainlike protease: a mysterious weapon. Journal of Biostatistics and Epidemiology, 2019. 5(4): p. 288-295.

19. Lei, X., et al., Activation and evasion of type I interferon responses by SARS-CoV-2. Nature communications, 2020. 11(1): p. 1-12.

20. Matthews, K., et al., The SARS coronavirus papain like protease can inhibit IRF3 at a post 
activation step that requires deubiquitination activity. Virology journal, 2014. 11(1): p. 209.

21. Li, S.-W., et al., SARS coronavirus papain-like protease inhibits the TLR7 signaling pathway through removing Lys63-linked polyubiquitination of TRAF3 and TRAF6. International journal of molecular sciences, 2016. 17(5): p. 678.

22. Sun, L., et al., Coronavirus papain-like proteases negatively regulate antiviral innate immune response through disruption of STING-mediated signaling. PloS one, 2012. 7(2): p. e30802.

23. Lowe, E.L., et al., Ubiquitination and deubiquitination: role in regulation of signaling by Toll-like receptors. Journal of endotoxin research, 2006. 12(6): p. 337-345.

24. Freitas, B.T., et al., Characterization and noncovalent inhibition of the deubiquitinase and deISGylase activity of SARS-CoV-2 papain-like protease. ACS Infectious Diseases, 2020. 6: p. 2099-2109.

25. Ratia, K., et al., A noncovalent class of papainlike protease/deubiquitinase inhibitors blocks SARS virus replication. Proceedings of the National Academy of Sciences, 2008. 105(42): p. 16119-16124.

26. Báez-Santos, Y.M., S.E.S. John, and A.D. Mesecar, The SARS-coronavirus papain-like protease: structure, function and inhibition by designed antiviral compounds. Antiviral research, 2015. 115: p. 21-38.

27. Lei, J., Y. Kusov, and R. Hilgenfeld, Nsp3 of coronaviruses: Structures and functions of a large multi-domain protein. Antiviral research, 2018. 149: p. 58-74.

28. Riva, L., et al., Discovery of SARS-CoV-2 antiviral drugs through large-scale compound repurposing. Nature, 2020. 586(7827): p. 113119.

29. Rut, W., et al., Activity profiling and crystal structures of inhibitor-bound SARS-CoV-2 papain-like protease: A framework for antiCOVID-19 drug design. Science advances, 2020. 6(42): p. eabd4596.

30. Klemm, T., et al., Mechanism and inhibition of the papain-like protease, PLpro, of SARSCoV-2. The EMBO journal, 2020. 39(18): p. e106275.

31. Shin, D., et al., Papain-like protease regulates SARS-CoV-2 viral spread and innate immunity. Nature, 2020. 587(7835): p. 657662.
32. Sun, M., et al., Inhibitors of RAS might be a good choice for the therapy of COVID-19 pneumonia. Zhonghua jie he he hu xi za zhi= Zhonghua jiehe he huxi zazhi= Chinese journal of tuberculosis and respiratory diseases, 2020. 43: p. E014-E014.

33. Phadke, M. and S. Saunik, Rapid response: Use of angiotensin receptor blockers such as Telmisartan, Losartsan in nCoV Wuhan Corona Virus infections-Novel mode of treatment. Response to the emerging novel coronavirus outbreak. BMJ, 2020. 368: p. $\mathrm{m} 406$.

34. Nejat, R. and A.S. Sadr, Are Losartan and Imatinib Effective Against SARS-CoV2 Pathogenesis? A Pathophysiologic-Based In Silico Study. In Silico Pharmacology, 2021. 9(1): p. 1.

35. Miesbach, W., Pathological role of angiotensin II in severe COVID-19. TH open: companion journal to thrombosis and haemostasis, 2020. 4(2): p. e138.

36. Wang, J., et al., The ACE2-deficient mouse: A model for a cytokine storm-driven inflammation. The FASEB Journal, 2020. 34(8): p. 10505-10515.

37. Rysz, S., et al., COVID-19 pathophysiology may be driven by an imbalance in the reninangiotensin-aldosterone system. Nature Communications, 2021. 12(1): p. 1-12.

38. Abd El-Aziz, T.M., A. Al-Sabi, and J.D. Stockand, Human recombinant soluble ACE2 (hrsACE2) shows promise for treating severe COVID19. Signal Transduction and Targeted Therapy, 2020. 5(1): p. 1-2.

39. Iwanaga, N., et al., Novel ACE2-IgG1 fusions with improved activity against SARS-CoV2, in bioRxiv. 2020.

40. Naito, T., et al., Angiotensin type 2 receptor actions contribute to angiotensin type 1 receptor blocker effects on kidney fibrosis. American Journal of Physiology-Renal Physiology, 2010. 298(3): p. F683-F691.

41. Tornling, G., et al., The angiotensin type 2 receptor agonist $C 21$ restores respiratory function in COVID19 - a double-blind, randomized, placebo-controlled Phase 2 trial. 2021: medRxiv.

42. Vaduganathan, M., et al., Renin-angiotensinaldosterone system inhibitors in patients with Covid-19. New England Journal of Medicine, 2020. 382(17): p. 1653-1659. 
43. Guo, J., et al., Coronavirus disease 2019 (covid-19) and cardiovascular disease: a viewpoint on the potential influence of angiotensin-converting enzyme inhibitors/angiotensin receptor blockers on onset and severity of severe acute respiratory syndrome coronavirus 2 infection. Journal of the American Heart Association, 2020. 9(7): p. $\mathrm{e} 016219$.

44. Otero, D.L., et al., Impact of angiotensinconverting enzyme inhibitors and angiotensin receptor blockers on COVID-19 in a western population. CARDIOVID registry. Revista Española de Cardiología (English Edition), 2020.

45. Koshy, A., et al., Renin-angiotensin system inhibition and risk of infection and mortality in COVID-19: a systematic review and metaanalysis. Internal Medicine Journal, 2020. 50(12): p. 1468-1474.

46. Elkahloun, A.G. and J.M. Saavedra, Candesartan could ameliorate the COVID-19 cytokine storm. Biomedicine \& Pharmacotherapy, 2020. 131: p. 110653.

47. Lukito, A.A., et al., Candesartan as a tentative treatment for COVID-19: a prospective nonrandomized open-label study. International journal of infectious diseases: IJID: official publication of the International Society for Infectious Diseases: p. S1201-9712 (21) 00420-3.

48. Duarte, M., et al., Telmisartan for treatment of Covid-19 patients: An open multicenter randomized clinical trial. EClinicalMedicine, 2021. 37: p. 100962.

49. Jin, F., Losartan, a selective antagonist of AT1 receptor, attenuates acute lung injury induced by seawater inhalation in rats. Chest, 2016. 149(4): p. A155.

50. Maveyraud, L. and L. Mourey, Protein X-ray Crystallography and Drug Discovery. Molecules, 2020. 25(5): p. 1030.

51. Kong, L., et al., Structural view and substrate specificity of papain-like protease from avian infectious bronchitis virus. Journal of Biological Chemistry, 2015. 290(11): p. 71607168.

52. Harcourt, J., et al., Severe acute respiratory syndrome coronavirus 2 from patient with coronavirus disease, United States. Emerging infectious diseases, 2020. 26(6): p. 1266.

53. Xu, J., Y. Zhang, and J.V. Williams, Development and optimization of a direct plaque assay for trypsin-dependent human metapneumovirus strains. Journal of virological methods, 2018. 259: p. 1-9.

54. Bakre, A., et al., Identification of host kinase genes required for influenza virus replication and the regulatory role of microRNAs. PloS one, 2013. 8(6): p. e66796.

55. Ratia, K., et al., Structural basis for the ubiquitin-linkage specificity and deISGylating activity of SARS-CoV papain-like protease. PLoS Pathog, 2014. 10(5): p. e1004113.

56. Hosseini-Zare, M.S., R. Thilagavathi, and C. Selvam, Targeting severe acute respiratory syndrome-coronavirus (SARS-CoV-1) with structurally diverse inhibitors: a comprehensive review. RSC Advances, 2020. 10(47): p. 28287-28299.

57. Sabarimurugan, S., et al., Comprehensive review on the prevailing COVID-19 therapeutics and the potential of repurposing SARS-CoV-1 candidate drugs to target SARSCoV-2 as a fast-track treatment and prevention option. Annals of translational medicine, 2020. 8(19).

58. Ratia, K., et al., Coronavirus Papain-like Peptidases. 3 ed. Handbook of Proteolytic Enzymes. Vol. 2. 2013: Academic Press. 2195-2199.

59. Gao, X., et al., Crystal structure of SARS-CoV2 papain-like protease. Acta Pharmaceutica Sinica B, 2020: p. 1-9.

60. Ren, X., et al., Analysis of ACE2 in polarized epithelial cells: surface expression and function as receptor for severe acute respiratory syndrome-associated coronavirus. Journal of general virology, 2006. 87(6): p. 1691-1695.

61. Ammerman, N.C., M. Beier-Sexton, and A.F. Azad, Growth and maintenance of Vero cell lines. Current protocols in microbiology, 2008. 11(1): p. A. 4E. 1-A. 4E. 7.

62. Todd, K.V. and R.A. Tripp, Vero Cells as a Mammalian Cell Substrate for Human Norovirus. Viruses, 2020. 12(4): p. 439.

63. Stoltz, M. and J. Klingström, Alpha/beta interferon $(I F N-\alpha / \beta)$-independent induction of IFN- $\lambda 1$ (interleukin-29) in response to Hantaan virus infection. Journal of virology, 2010. 84(18): p. 9140-9148.

64. Desmyter, J., J.L. Melnick, and W.E. Rawls, Defectiveness of interferon production and of rubella virus interference in a line of African 
green monkey kidney cells (Vero). Journal of virology, 1968. 2(10): p. 955-961.

65. Ng, M.-L., et al., Proliferative growth of SARS coronavirus in Vero E6 cells. Journal of General Virology, 2003. 84(12): p. 32913303.

66. Cox, R.M., J.D. Wolf, and R.K. Plemper, Therapeutically administered ribonucleoside analogue MK-4482/EIDD-2801 blocks SARS-
CoV-2 transmission in ferrets. Nature microbiology, 2020: p. 1-8.

67. Yoon, J.-J., et al., Orally efficacious broadspectrum ribonucleoside analog inhibitor of influenza and respiratory syncytial viruses. Antimicrobial agents and chemotherapy, 2018. 62(8). 